\title{
Nonlinear Intestinal Absorption of Fluorescein Isothiocyanate Dextran 4000 Caused by Absorptive and Secretory Transporting System
}

\author{
Mikio Tomita, Rie Ohkubo, Shohei Ouchi, Chise Kawahata, Masahiro Hayashi
}

Department of Drug Absorption and Pharmacokinetics, School of Pharmacy, Tokyo University of Pharmacy and Life Sciences, Tokyo, Japan.

Email: tomita@toyaku.ac.jp

Received April $5^{\text {th }}, 2011$; revised May $2^{\text {nd }}, 2012$; accepted June $6^{\text {th }}, 2011$.

\begin{abstract}
The mechanism of the nonlinear concentration dependence of the intestinal absorption of fluorescein isothiocyanate dextran 4,000 (FD-4) was studied using in situ rat intestinal loops and the in vitro Ussing-type chamber method. The intestinal absorption rate constant of FD-4, as evaluated by the intestinal loop method, increased significantly in a nonlinear fashion as the FD-4 concentration increased up to $0.2 \mathrm{mM}$ and tended to decrease at concentrations higher than $0.2 \mathrm{mM}$. The mucosal-to-serosal permeation of FD-4 across rat ileal sheets, as evaluated by the in vitro Ussing-type chamber method, also increased in a nonlinear fashion in the low concentration range (0.01- $0.02 \mathrm{mM})$, before decreasing as the concentration increased further, whereas serosal-to-mucosal permeation decreased in a concentration-dependent manner. In addition, mucosal-to-serosal flux and serosal-to-mucosal flux were increased and reduced in the presence of the metabolic inhibitor 2,4-dinitrophenol, respectively. These results suggest that FD-4 is predominantly secreted into the intestinal lumen by an efflux transport system.
\end{abstract}

Keywords: Fluorescein Isothiocyanate Dextran 4000, Nonlinear Absorptive Transport, Nonlinear Secretory Transport, Rat Ileal Intestine

\section{Introduction}

Under normal conditions, the intestinal epithelium acts as a selective barrier that defines and maintains distinctive luminal and subepithelial compartments. The barrier function of the intestine permits the systemic absorption of nutrients while it prevents systemic contamination by luminal microbes or microbial products [1]. In both clinical and experimental studies, the functional integrity of the intestinal epithelial barrier has often been assessed by measuring the mucosal permeability of certain hydrophilic compounds, such as ${ }^{51} \mathrm{Cr}$-EDTA [2], lactulose [3], cellobiose [4], polyethylene glycols [5], fluorescent dyes [6], and fluorescein isothiocyanate (FITC)-labeled dextrans [7]. It is generally accepted that the transepithelial movement of these hydrophilic probes occurs as a result of passive diffusion through the paracellular channels formed by adjacent enterocytes. The rate of transepithelial permeation is thought to be regulated by the tight junctions guarding the apical end of paracellular pores [8] as well as the hydrodynamic size of the hydrophilic probes used for the measurements [9]. In some in vivo experimental studies, permeability was measured in the lumen-to-plasma direction [5]; whereas in others, permeability was measured in the plasma-to-lumen direction [7], and both types of studies were performed under the assumption that directional permeation involves passive diffusion and is not polarized.

However, it has become apparent that many substances are actively transported across intestinal epithelia. For example, it has been reported that poorly absorbed $\beta$ actam antibiotics, such as cefazolin and ampicillin, are actively secreted in the serosal-to-mucosal direction by one or more mechanisms that are inhibited by both organic anions and some organic cations. The multidrug resistance (MDR) gene product, which is a $170-\mathrm{kD}$ plasma membrane glycoprotein and is commonly designated Gp170 or P-glycoprotein (P-gp), has been implicated in the active intestinal secretion of various hydrophobic or amphipathic molecules, including cyclosporine A, daunomycin, rhodamine 123 , and some small molecular pep- 
tides.

On the other hand, we have demonstrated that the secretion of fluorescein isothiocyanate dextran 4,000 (FD4; MW 4,400 Da), which is considered to be an important paracellular probe for assessing transport in the serosal to mucosal direction across rat colonic epithelial cells and Caco-2 cell monolayers, is mediated by transcytosis mechanisms, one of which shows substrate specificity for dextran polysaccharides $[10,11]$. Thus, the absorption mechanism of FD-4 is complex and needs to be studied further.

In the present study, we investigated the absorptive and secretory transport of FD-4 in the rat intestine and revealed the participation of multiple transport mechanisms.

\section{Materials and Methods}

\subsection{Materials}

Fluorescein isothiocyanate dextran 4,000 (FD-4); fluorescein isothiocyanate dextran 40,000 (FD-40); 2,4-dinitrophenol (DNP); colchicine; chloroquine; methyl- $\beta$ cyclodextrin; fucoidan; polyinosinic acid (5') potassium salt (poly (I)); cyclosporine A; probenecid; and glutathione (GSH) were purchased from Sigma (St. Louis). All other chemicals were commercial products of reagent grade.

\subsection{Measurement of Intestinal Absorption by the in Situ Loop Method Materials}

The intestinal absorption of FD-4 was evaluated using the loop method $[12,13]$. The ileal of male Wistar/ST rats weighing $200 \mathrm{~g}$ to $250 \mathrm{~g}$ (Japan SLC, Hamamatsu, Japan) were exposed by making an abdominal incision along the midline, and two L-shaped glass cannulae (i.d.: $2 \mathrm{~mm}$, o.d.: $4 \mathrm{~mm}$ ) were inserted through small slits at the proximal and distal ends $(7 \mathrm{~cm})$. The proximal and distal cannulae were located at $12 \mathrm{~cm}$ and $5 \mathrm{~cm}$ above the cecum, respectively. Each cannula was secured by ligation with a silk suture, and the intestine was returned to the abdominal cavity to maintain its integrity. A 4-cm portion of Tygon tubing (i.d.: $3 \mathrm{~mm}$, o.d.: $5 \mathrm{~mm}$ ) was attached to the exposed end of each cannula, and a 10-ml hypodermic syringe fitted with a connecting tube and containing loop solution pre-warmed at $37^{\circ} \mathrm{C}$ was attached to the proximal cannula. To clear the gut, saline was slowly passed through it to the distal cannula and discarded until the effluent was clear. The remaining loop solution was carefully expelled from the intestine by pumping air through the syringe, and $5 \mathrm{ml}$ of FD-4 solution were immediately introduced into the intestine. The distal cannula was connected to a $10-\mathrm{ml}$ syringe fit- ted with a three-way stopcock. At 15, 30, 45, and 60 min after the administration of the drug solution, a $0.5-\mathrm{ml}$ aliquot of luminal solution was removed through the attached syringe. The FD-4-containing test solution was composed of $126 \mathrm{mM} \mathrm{NaCl}, 5.0 \mathrm{mM} \mathrm{KCl}, 1.4 \mathrm{mM}$ $\mathrm{CaCl}_{2}, 3.5 \mathrm{mM} \mathrm{NaHCO}_{3}, 4.85 \mathrm{mM} \mathrm{NaH} \mathrm{PO}_{4} \cdot 2 \mathrm{H}_{2} \mathrm{O}, 0.95$ $\mathrm{mM} \mathrm{Na} 2 \mathrm{HPO}_{4}$, and $2 \mathrm{~g} / \mathrm{L} \mathrm{D} \mathrm{(+)-glucose} \mathrm{at} \mathrm{pH} 6.5$, and the solution was gassed with $95 \% \mathrm{O}_{2} / 5 \% \mathrm{CO}_{2}$ before and during the transport experiment. The change in the volume of water in the intestinal luminal solution was corrected for by measuring the change in the concentration of the unabsorbable marker fluorescein isothiocyanate dextran 40,000 (FD-40), which was administered simultaneously without FD-4. The concentration of FD-40 used in this study was $0.1 \%$.

\subsection{Transport Experiments Involving the Ussing-Type Chamber Method}

Rat ileal tissue sheets were prepared as described previously [14]. Tissue sheets consisting of the mucosa and most of the muscularis mucosa were prepared by removing the submucosa and tunica muscularis with fine forceps. They were then mounted vertically in an Ussingtype chamber that provided an exposed area of $0.75 \mathrm{~cm}^{2}$. The volume of bathing solution on each side was $11 \mathrm{ml}$, and the solution temperature was maintained at $37^{\circ} \mathrm{C}$ in a water-jacketed reservoir. The FD-4-containing test solution was composed of $126 \mathrm{mM} \mathrm{NaCl}, 5.0 \mathrm{mM} \mathrm{KCl}, 1.4$ $\mathrm{mM} \mathrm{CaCl}_{2}, 3.5 \mathrm{mM} \mathrm{NaHCO}_{3}, 4.85 \mathrm{mM} \mathrm{NaH} \mathrm{PO}_{4} \cdot 2 \mathrm{H}_{2} \mathrm{O}$, $0.95 \mathrm{mM} \mathrm{Na}_{2} \mathrm{HPO}_{4}$, and $2 \mathrm{~g} / \mathrm{L} \mathrm{D}(+)$-glucose at $\mathrm{pH} 6.5$, and the solution was gassed with $95 \% \mathrm{O}_{2} / 5 \% \mathrm{CO}_{2}$ before and during the transport experiment. To examine the effects of 2,4-dinitrophenol (DNP), a metabolic inhibitor, and colchicines [15], chloroquine [16], methyl- $\beta$-cyclodextrin [17], fucoidan [18], poly (I) [19], cyclosporine A [20], and probenecid +GSH [21], which inhibit various transport systems, the mucosal reservoir was filled with test solution containing one of the above inhibitors. The $\mathrm{pH}$ of the test solution containing DNP was adjusted to 6.5 using sodium hydroxide. Samples were taken from the acceptor side at intervals of $10 \mathrm{~min}$.

\subsection{Assay and Data Analysis}

The concentration of FD-4 was determined using a fluorescence spectrophotometer after appropriate dilution of the samples in phosphate-buffered saline ( $\mathrm{pH} 7.4)$. The excitation and emission wavelengths of FD-4 were 492 and $515 \mathrm{~nm}$, respectively.

The absorption rate constant was evaluated from the slope of the decline in the FD-4 concentration of the luminal fluid over time.

Permeation clearance was obtained as follows: 
Permeation clearance $=(\mathrm{dQ} / \mathrm{dt}) /\left(\mathrm{A} \times \mathrm{C}_{0}\right)$,

where $\mathrm{dQ} / \mathrm{dt}$ is the transport rate $(\mu \mathrm{g} / \mathrm{min})$ and corresponds to the slope of the linear regression line between the amount transported and time. $\mathrm{C}_{0}$ is the initial concentration in the donor chamber $(\mu \mathrm{g} / \mathrm{mL})$, and $\mathrm{A}$ is the area of the membrane $\left(0.75 \mathrm{~cm}^{2}\right)$.

\subsection{Statistical Analysis}

All results are expressed as the mean value \pm standard error (Mean \pm S.E.). The statistical significance of differences between two groups was analyzed using Dunnett's test. Differences were considered to be significant at a level of $p<0.05$.

\section{Results}

\subsection{Concentration Dependence of the Intestinal Absorption Rate of FD-4 in Rats}

The relationship between the first-order absorption rate constant and the FD-4 concentration was evaluated by the loop method. When 0.01 to $1 \mathrm{mM}$ FD-4 was administered to the ileal loop, the intestinal absorption rate changed nonlinearly, as shown in Figure 1. The intestinal absorption rate increased from $0.01 \mathrm{mM}$ to $0.2 \mathrm{mM}$, and then decreased from $0.2 \mathrm{mM}$ to $1 \mathrm{mM}$. The absorption rate constant at $0.2 \mathrm{mM}$ was significantly higher than that at 0.01 or $0.02 \mathrm{mM}(p<0.05)$. This result suggests that at least two nonlinear events are involved in

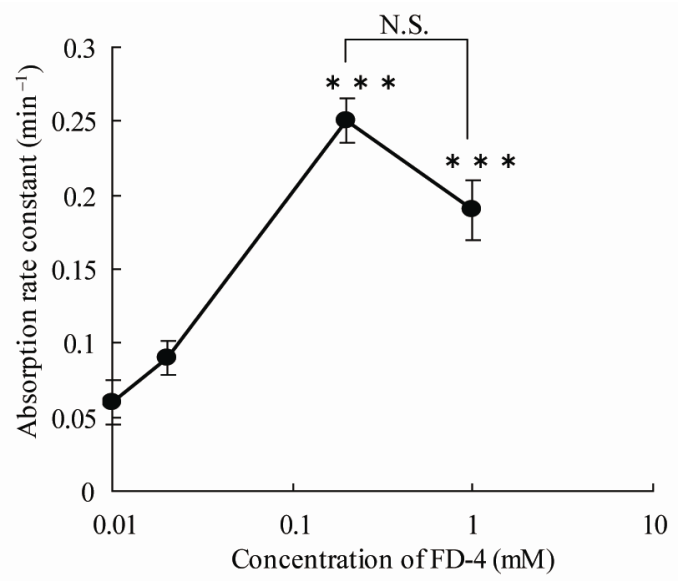

Figure 1. Concentration dependence of the FD-4 ileal absorption rate constant in rats. The intestinal absorption rate constant (ka) of FD-4 (0.01 mM - $1 \mathrm{mM}$ ) was evaluated throughout the time course of the decrease in the luminal FD-4 concentration measured by the in situ loop method; Each point represents the mean $\pm S$.E. of seven experiments. ${ }^{* * *}$ Significantly different from the absorption rate constant observed at $0.01 \mu \mathrm{M}$ FD-4 $(p<0.001)$. N.S. not significantly different from the absorption rate constant measured at 0.2 mM FD-4. the I testinal absorption of FD-4.

The transport of FD-4 in rat intestinal tissue was examined further using the diffusion chamber method. The permeation of FD-4 at various concentrations across a rat ileal sheet was measured in both the mucosal-to-serosal and serosal-to-mucosal directions. As shown in Figure 2, the serosal-to-mucosal permeation coefficient significantly decreased in a concentration-dependent manner, whereas the mucosal-to-serosal permeation coefficient increased up to $0.02 \mathrm{mM}$, and then tended to decrease at concentrations higher than $0.02 \mathrm{mM}$. Furthermore, in the presence of $1 \mathrm{mM}$ DNP, the serosal-to-mucosal transport clearance of $0.01 \mathrm{mM}$ FD-4 significantly decreased, whereas transport in the reverse direction increased significantly (Figure 2). At FD-4 concentrations above 0.02 $\mathrm{mM}$, no vectorial transport of FD-4 across the rat ileal membrane was observed (Figure 2), and the effect of DNP disappeared (data not shown)

\subsection{Transcellular Transport of FD-4 across a Rat Ileal Sheet}

To determine whether the transport of FD-4 across a ratileal sheet was temperature dependent, transport fluxes

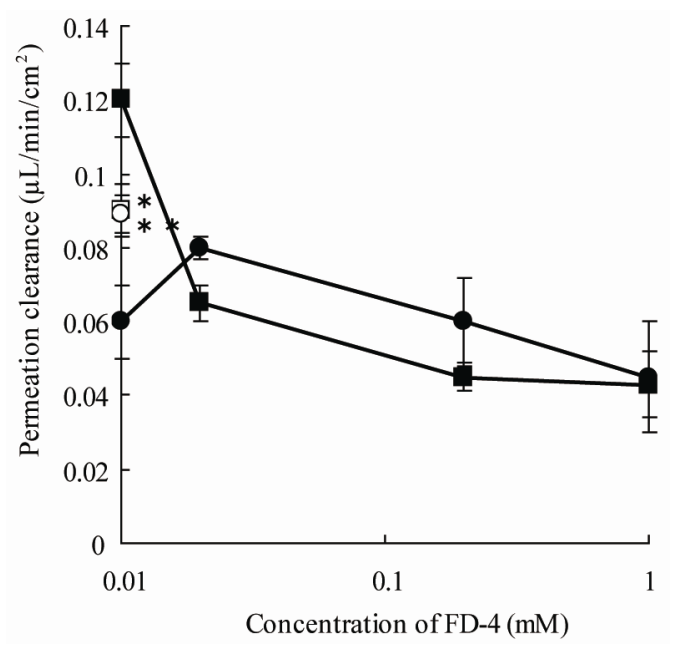

Figure 2. Concentration dependence of FD-4 permeation clearance across rat ileal tissue. The permeation of FD-4 was determined using a rat ileum mounted in an Ussingtype chamber. The closed squares and closed circles represent the permeation clearance in the serosal-to-mucosal and mucosal-to-serosal directions, respectively. The open squares and open circles represent the serosal-to-mucosal and mucosal-to serosal clearance of FD-4 in the presence of $1 \mathrm{mM}$ 2,4-dinitrophenol. Each point represents the mean \pm S.E. of seven experiments. Significantly different from the mucosal-to-serosal clearance in the absence of 2,4-dinitrophenol $(p<0.05) .{ }^{* *}$ Significantly different from the serosal-to-mucosal clearance in the absence of 2,4-dinitrophenol $(p<0.01)$. 
were measured at $4^{\circ} \mathrm{C}$ and $37^{\circ} \mathrm{C}$ (Figure 3). The permeation of FD-4 was linear over 120 min after an initial lag time of a few minutes. The flux in the serosal-to mucosal direction was about 2.0 - 3.3 times larger than the reverse flux (Figure 3), and the flux observed at $4^{\circ} \mathrm{C}$, which is regarded as a measure of passive permeability, was significantly lower than that seen at $37^{\circ} \mathrm{C}$. Therefore, the transport of FD-4 can be ascribed to active transcellular permeation.

To investigate whether FD-4 interacts with receptors or transporters present in rat ileal epithelial cells, the effects of colchicines [15], chloroquine [16], methyl- $\beta$ cyclodertin [17], fucoidan [18], poly(I) [19], cyclosporine A [20], and probenecid + GSH [21] on the serosal to mucosal transport of FD-4 were examined. When colchicines, a typical inhibitor of transcytosis including endocytosis and exocytosis, was used at a concentration of 2 $\mathrm{mM}$, the transport of FD-4 in the serosal-to-mucosal direction decreased (Figure 4), while that of FITC without dextran was not decreased (Figure 5). When Cyclosporine A, a typical substrate for P-gp, was added at a concentration that was 2 times higher than that of FD-4, the transport of FD-4 was significantly decreased, and it was also decreased in the presence of $\mathrm{ABC}$ transport inhibitors such as a probenecid + GSH and various other endocytosis inhibitors such as chloroquine, methyl- $\beta$ cyclodextrin, and fucoidan (Figure 4). However, no marked changes in the serosal-to-mucosal transport of FITC were observed in the presence or absence of pro-

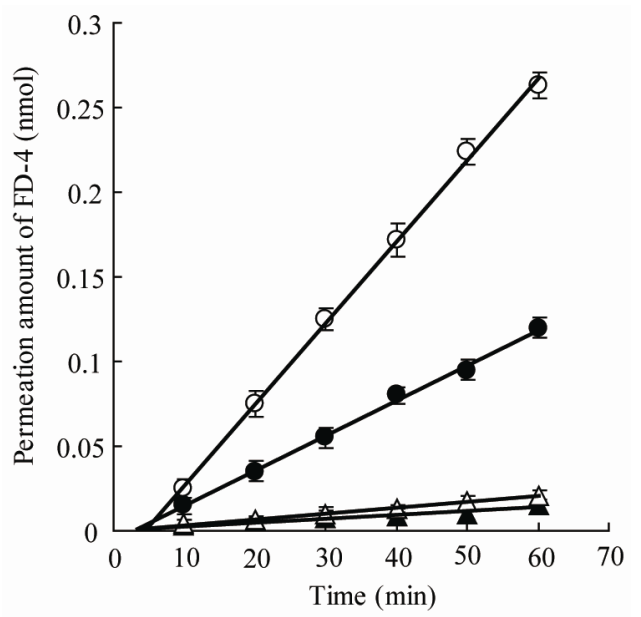

Figure 3. Effect of temperature on the polarized permeation of FD-4 across rat ileal tissue. The concentration of FD-4 used in this study was $10 \mu \mathrm{M}$. The circles and triangles represent the data obtained at $37^{\circ} \mathrm{C}$ and $4^{\circ} \mathrm{C}$, respectively. The white and black symbols represent the data for the serosal-to-mucosal and mucosal-to-serosal directions, respectively. Each point represents the mean \pm S.E. of seven experiments.

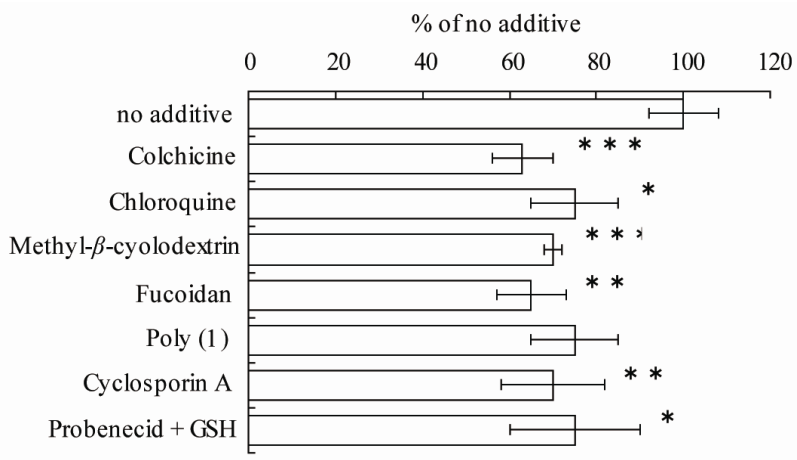

Figure 4. Effects of various inhibitors on the serosal to mucosal transport of FD-4 across rat ileal tissue. The concentrations of colchicines, chloroquine, methyl- $\beta$-cyclodextrin, fucoidan, poly (I), cyclosporine A, probenecid, and GSH used in the present study were $2 \mathrm{mM}, 0.1 \mathrm{mM}, 5 \mathrm{mM}, 0.2$ $\mathrm{mg} / \mathrm{ml}, 50 \mu \mathrm{g} / \mathrm{ml}, 20 \mu \mathrm{M}, 1 \mathrm{mM}$, and $10 \mathrm{mM}$, respectively. Each point represents the mean \pm S.E. of seven experiments; ${ }^{*}(p<0.05) ;{ }^{* *}(p<0.01) ;{ }^{* * *}(p<0.001)$, significantly different from the data obtained without the inhibitor (no additive).

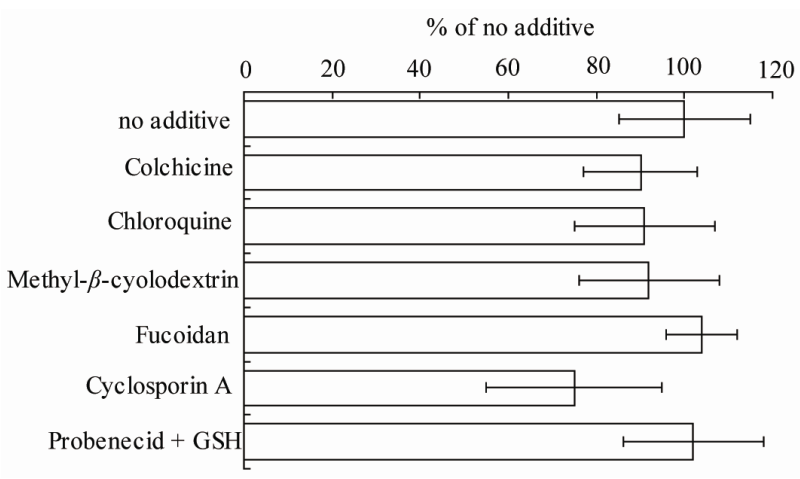

Figure 5. Effects of various inhibitors on the serosal to mucosal transport of FITC across rat ileal tissue. The concentrations of colchicines, chloroquine, methyl-b-cyclodextrin, fucoidan, poly (I), cyclosporine A, probenecid, and GSH used in the present study were $2 \mathrm{mM}, 0.1 \mathrm{mM}, 5 \mathrm{mM}, 0.2$ $\mathrm{mg} / \mathrm{ml}, 50 \mu \mathrm{g} / \mathrm{ml}, 20 \mu \mathrm{M}, 1 \mathrm{mM}$, and $10 \mathrm{mM}$, respectively. Each point represents the mean \pm S.E. of seven experiments.

benecid $+\mathrm{GSH}$ (Figure 5). Chloroquine, methyl- $\beta$-cyclodextrin, fucoidan, and cyclosporine A had no significant effect on FITC transport (Figure 5).

\section{Discussion}

The concept that carrier-mediated intestinal absorption and luminal secretion mechanisms as well as intestinal tissue metabolic activity regulate the bioavailability of various drugs has been established previously [22,23]. Such saturable physiological mechanisms can sometimes produce nonlinear pharmacokinetic phenomena. The re- 
sults obtained in the present study represent the first evidence that a complex pattern of nonlinear FD-4 bioavailability is generated by the interaction of intestinal absorptive and secretory transport systems when FD-4 is present at a concentration beyond that which induces linear absorbability in the intestinal luminal fluid.

The intestinal absorption of FD-4 in rats, as assessed by the in situ loop method, exhibited distinctive nonlinearity, involving a significant increase of the first-order absorption rate constant at a drug concentration of 0.2 $\mathrm{mM}$ compared with those observed at 0.01 and $0.02 \mathrm{mM}$, followed by a decrease at higher concentrations $(1 \mathrm{mM})$ (Figure 1). As the disappearance of intact FD-4 from the intestinal luminal fluid was measured in this experiment, the above observations can be accounted for by saturable secretory and absorptive transport mechanisms as these phenomena can not be induced by metabolism saturation or a solubility limitation. Absorptive-directed (mucosalto-serosal) flux of FD-4 across ileal tissue preparations mounted on an Ussing-type chamber increased with the concentration of the drug up to $0.02 \mathrm{mM}$ (Figure 2). This result is consistent with that obtained by the in situ loop method, both of which can be explained by a saturable secretory mechanism. This hypothesis is further supported by our measurements of serosal-to-mucosal flux using the Ussing-type chamber method, which showed a marked decrease in the permeation coefficient as the FD-4 concentration increased (Figure 2). The break points shown in Figures 1 and $\mathbf{2}$ occurred at 0.2 $\mathrm{mM}$ and $0.02 \mathrm{mM}$, respectively. This concentration difference can be ascribed to the difference in the thickness of the unstirred water layer between these two experimental systems because the loop method is expected to have a thicker unstirred water layer than the isolated ileal sheet chamber method. Conversely, in the in vitro system, the solution in contact with the tissue was stirred at $95 \% \mathrm{O}_{2} / 5 \% \mathrm{CO}_{2}$ throughout the experiment.

The vectorial transport of FD-4 across a rat ileal sheet displayed a higher permeation constant in the serosal-tomucosal direction than in the reverse direction (Figure 3). Accordingly, concentration-dependent and vectorial transport may operate during rat intestinal absorption. Here, the mucosal-to-serosal flux of FD-4 at $37^{\circ} \mathrm{C}$ was significantly higher than that at $4^{\circ} \mathrm{C}$ (Figure 3), supporting the active transport of FD-4 across rat ileal sheets.

In a previous study using an Ussing-type chamber, we showed that the permeability of laminaran, a $(1 \rightarrow 3)-\beta$ D-glucan used as a water soluble and high molecular weight probe, is greater in the serosal-to-mucosal direction than the mucosal-to-serosal direction [24]. Laminaran is a dextran polysaccharide that consists of repeating D-glucose units connected by $\alpha$-glycoside linkages. The polarized serosal-to-mucosal flux of FD-4 but not Lucifer yellow (a substrate of fluid-phase endocytosis) was inhibited when excess dextran 10,000, a structurally similar polysaccharide, was added to the FD-4 solution [25]. These findings suggest that at least two distinct polarized transport systems exist, one of which shows some degree of substrate specificity for dextrans. We also considered the possibility that the polarized transport of FD-4 and laminaran was mediated by membrane traffic such as fluid-phase endocytosis. This notion is not a novel concept. Using primary cultures of canine proximal tubular renal epithelial cells, Goligorsky et al. reported that the flux of LY, which is generally regarded as a marker of fluid-phase endocytosis, was 3-fold greater in the basal-to-apical direction than in the apical-to-basal direction [26]. In contrast, Pantzar et al. reported that the net rate of fluid-phase transcytosis of $\left[{ }^{3} \mathrm{H}\right]$ inulin across cultured MDCK epithelial cells was approximately equal in both directions, even though the basolateral endocytotic rate was 6-fold greater than the apical rate [27]. In our study, we observed that the mucosa-to-serosal and serosal-to-mucosal fluxes of FD-4 involve nonlinear transport that is dependent on the concentration of FD-4 in the donor chamber. These findings are consistent with transport in both directions occurring as a result of membrane traffic and paracellular permeation, or a combination of both processes. Our results are insufficient to entirely exclude the possibility that polarized fluid phase transcytosis (or some other nonsaturable process) contributes to the vectorial transport of FD-4.

In conclusion, fluorescein isothiocyanate dextran 4,000 (FD-4), displayed nonlinear intestinal absorption involving increases at lower concentrations followed by decreases at higher concentrations. Such nonlinearity can be explained by the operation of absorptive and secretory transporters or other mechanisms in the intestine, although no specific secretory or absorptive transporters have been identified yet. Clarification of the mechanistic and kinetic features of the nonlinear intestinal absorption of FD-4 is important to aid our understanding of the systems used to transport high molecular and hydrophilic compounds in humans.

\section{Acknowledgments}

This research was supported partly by a Grant-in-Aid for Scientific Research (No. 21590182) from the Ministry of Education, Science, and Culture of Japan and by grants from the Japan Health Sciences Foundation.

\section{References}

[1] J. S. Aranow and M. P. Fink, "Determinant of Intestinal Barrier Failure in Critical Illness," British Journal of An- 
esthesia, Vol. 77, 1996, pp. 71-81.

[2] D. W. Riddington, B. Venkatesh, C. M. Boivin, et al., "Intestinal Permeability, Gastric Intramucosal pH, and Systemic Endotoxemia in Patients Undergoing Cardiopulmonaly Bypass," The Journal of the American Medical Association, Vol. 275, 1996, pp. 1007-1012. doi:10.1001/jama.275.13.1007

[3] P. L. Faries, R. J. Simon, A. T. Martella, et al., "Intestinal Permeability Correlates with Severity of Injury in Trauma Patients," The Journal of Trauma, Vol. 44, No. 6, 1998, pp. 1031-1036. doi:10.1097/00005373-199806000-00016

[4] H. M. Oudemans van Straaten, P. G. Jansen, F. J. Hoek, et al., "Intestinal Permeability, Circulating Endotoxin, and Postoperative Systemic Responses in Cardiac Surgery Patients," Journal of Cardiothoracic and Vascular Anesthesia, Vol. 10, 1996, pp. 187-194. doi:10.1097/00005373-199806000-00016

[5] R. Schleiffer and F. Raul, "Prophylactic Administration of L-Arginine Improves the Intestinal Barrier Function after Mesenteric Ischemia," Gut, Vol. 39, No. 2, 1996, pp. 194-198. doi:10.1097/00005373-199806000-00016

[6] A. L. Salzman, P. S. Wollert, H. Wang, et al., "Intraluminal Oxygenation Ameliorates Ischemia/Reperfusion-inDuced Gut Mucosal Hyperpermeability in Pigs," Circulatory Shock, Vol. 40, No. 1, 1993, pp. 37-46.

[7] A. L. Salzman, H. Wang, P. S. Wollert, et al., "Endotoxin-Induced Ileal Mucosal Hyperpermeability in Pigs: Role of Tissue Acidosis," American Journal of Physiology, Vol. 266, No. 4, 1994, pp. G633-G646.

[8] J. L. Madara, "Loosening Tight Junctions: Lessons from the Intestine," The Journal of Clinical Investgation, Vol. 83, No. 4, 1989, pp. 1089-1094. doi:10.1172/JCI113987

[9] T. Sawada, T. Ogawa, M. Tomita, et al., "Role of Paracellular Pathway in Nonelectrolyte Permeation across Rat Colon Epithelium Enhanced by Sodium Caprate and Sosium Caprylate," Pharmaceutical Research, Vol. 8, No. 11, 1991, pp. 1365-1371. doi:10.1023/A:1015840921203

[10] M. Tomita, Y. Hotta, R. Ohkubo and S. Awazu, "Polarized Transport Wa Observed not in Hydrophilic Compounds but in Dextran in Caco-2 cell Monolayers," Biological \& Pharmaceutical Bulletin, Vol. 22, 1999, pp. 330-331.

[11] M. Tomita, M. J. Menconi, R. L. Delude and M. P. Fink., "Polarized Transport of Hydrophilic Compounds across Rat Colonic Mucosa from Serosa to Mucosa is Temperature Dependent," Gastroenterology, Vol. 118, No. 3, 2000 , pp. $535-543$. doi:10.1016/S0016-5085(00)70259-9

[12] A. Iida, M. Tomita, Y. Matsuura, et al., "Improvement of Intestinal Absorption of P-Glycoprotein Substrate by D-Tartaric Acid," Drug Metabolism and Pharmacokinetics, Vol. 21, No. 5, 2006, pp. 424-428. doi: $10.2133 / \mathrm{dmpk} .21 .424$

[13] J. T. Doluisio, N. F. Billups, E. T. Sugita, et al., "Drug Absorption I: An in Situ Rat Gut Technique Yielding Realistic Absorption Rates," Journal of Pharmaceutical
Sciences, Vol. 58, No. 10, 1969, pp. 1196-1202. doi:10.1002/jps.2600581006

[14] M. Tomita, R. Ohkubo and M. Hayashi, "Lipopolysaccharide Transport System across Colonic Epithelial Cells in Normal and Infective Rat," Drug Metabolism and Pharmacokinetics, Vol. 19, No. 1, 2004, pp. 33-40. doi: $10.2133 / \mathrm{dmpk} .19 .33$

[15] K. Sandvig and B. van Deurs, "Selective Modification of the Endocytic Uptake of Eicin and Fluid Phase Markers without Alteration in Transferring Endocytosis," The Journal of Biological Chemistry, Vol. 265, 1990, pp. 6382-6388.

[16] S. Wu-Pong, T. L. Weiss and C. A. Hunt, "Antisense Cmyc Oligodeoxynucleotide Cellular Uptake," Pharmaceutical Research, Vol. 9, No. 8, 1992, pp. 1010-1017. doi:10.1023/A:1015846209681

[17] S. K. Rodal, G. Skretting, O. Garred, et al., "Extraction of Cholesterol with Methyl- $\beta$-Cyclodextrin Perturbs Formation of Clathrin-Coated Endocytic Vesicles," Molecular Biology of the Cell, Vol. 10, No. 4, 1999, pp. 961-974.

[18] D. J. Falcone, "Heparin Stimulation of Plasminogen Activator Secretion by Macrophage-Like Cell Line RAW264.7: Role of the Scavenger Receptor," Journal of Cellular Physiology, Vol. 140, No. 2, 1989, pp. 219-226. doi:10.1023/A:1015846209681

[19] L. Rohrer, M. Freeman, T. Kodama, et al., "Coiled Coli fibrous Domains Mediated Ligand Binding by Macrophage Scavenger Receptor Type II," Nature, Vol. 343, 1990, pp. 570-572. doi:10.1038/343570a0

[20] B. L. Leu and J. D. Huang, "Inhibition of Intestinal PGlycoprotein and Effect on Etoposide Absorption," Cancer Chemotherapy and Pharmacology, Vol. 35, No. 5, 1995, pp. 432-436. doi:10.1007/s002800050258

[21] G. Rappa, A. Lorico, R. A. Flavell, et al., "Evidence that the Multidrug Resistance Protein (MRP) Function as a Co-transporter of Glutathione and Natural Product Toxins," Cancer Research, Vol. 57, No. 23, 1997, pp. 52325237.

[22] I. Tamai, A. Saheki, R. Saitoh, et al., "Nonlinear Intestinal Absorption of 5-Hydroxytrip-Tamine Receptor Antagonist Caused by Absorptive and Secterory Transporters," The Journal of Pharmacology and Experimental Therapeutics, Vol. 283, No. 1, 1997, pp. 108-115.

[23] L. Z. Benet, C. Y. Wu, M. F. Hebert, et al., "Intestinal Drug Metabolism and Antiport Processes: A Potential Paradigm I Oral Drug Delivery," Journal of Controlled Release, Vol. 39, 1996, pp. 139-143. doi:10.1016/0168-3659(95)00147-6

[24] M. Tomita, M. Miwa, S. Ohuchi, et al., "Nonlinear Intestinal Absorption of $(1 \rightarrow 3) \beta$-D-Glucan Caused by Absorptive and Secretory Transporting System," Biological \& Pharmaceutical Bulletin, Vol. 32, No. 7, 2009, pp. 1295-1297. doi:10.1248/bpb.32.1295

[25] M. Tomita, M. J. Menconi, R. L. Dekude, et al., "Polarized Transport of Hydrophilic Compounds across Rat Colonic Mucosa from Serosa to Mucosa is Temperature 
Dependent," Gastroenterology, Vol. 118, No. 3, 2000, pp. 535-543. doi:10.1016/S0016-5085(00)70259-9

[26] M. S. Goligorsky and K. A. Hruska, "Transcytosis in Cultured Proximal Tubular Cells," Journal of Membrane Biology, Vol. 93, No. 3, 1986, pp. 237-247. doi:10.1007/BF01871178
[27] N. Pantzar, S. Lundin and B. R. Westrom, "Bidirectional Small-Intestinal Permeability in the Rat to Some Common Marker Molecules in Vitro," Scandinavian Journal of Gastroenterology, Vol. 29, 1994, pp. 703-709. doi:10.3109/00365529409092497 